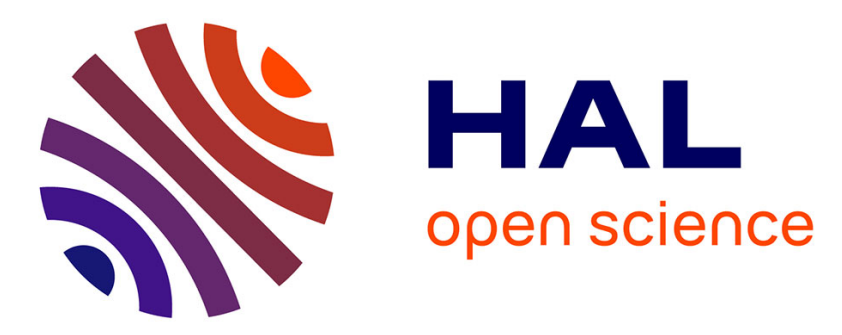

\title{
Influence of intrinsic strain on irradiation induced damage: the role of threshold displacement and surface binding energies
}

\author{
Julien Guénolé, A. Prakash, E. Bitzek
}

\section{- To cite this version:}

Julien Guénolé, A. Prakash, E. Bitzek. Influence of intrinsic strain on irradiation induced damage: the role of threshold displacement and surface binding energies. Materials \& Design, 2016, 111, pp.405-413. 10.1016/j.matdes.2016.08.077 . hal-02459274

\section{HAL Id: hal-02459274 \\ https://hal.science/hal-02459274}

Submitted on 7 May 2020

HAL is a multi-disciplinary open access archive for the deposit and dissemination of scientific research documents, whether they are published or not. The documents may come from teaching and research institutions in France or abroad, or from public or private research centers.
L'archive ouverte pluridisciplinaire HAL, est destinée au dépôt et à la diffusion de documents scientifiques de niveau recherche, publiés ou non, émanant des établissements d'enseignement et de recherche français ou étrangers, des laboratoires publics ou privés. 


\title{
Influence of intrinsic strain on irradiation induced damage: the role of threshold displacement and surface binding energies
}

\author{
J. Guénolé*, A. Prakash, E. Bitzek \\ Department of Materials Science and Engineering, Institute I, \\ Friedrich-Alexander-Universität Erlangen-Nürnberg (FAU), 91058 Erlangen, Germany
}

\begin{abstract}
Focused ion beam (FIB) machining has become a standard tool for sample preparation and in combination with digital image correlation (DIC) for the evaluation of local intrinsic stresses by measuring strain relaxation. However, FIB milling always leads to irradiation damage of the material. Current models for the formation of irradiation damage and the sputter yield are based on two key parameters, the threshold displacement energy (TDE) and surface binding energy (SBE), which are usually determined from unstrained systems with idealized surfaces. Here we use atomistic simulations to determine the TDE and SBE for strained silicon and aluminum and compare the results to full cascade simulations. A clear, material class dependent influence of the strain state on the TDE is observed, and surface amorphisation is shown to significantly increase the SBE of $\left\{\begin{array}{lll}0 & 0 & 1\end{array}\right\}$ surfaces.
\end{abstract}

Keywords: FIB, irradiation damage, atomistic simulation, threshold displacement energy, surface binding energy

\section{Introduction}

Over the past few decades, the focused ion beam (FIB) technique has established itself in the material science community as an indespensible tool in the field of micro/nano mechanics [1, 2], with typical applications including sample preparation for electron microscopy [3] or atom probe tomography [4] as well as

\footnotetext{
* Corresponding author

Email address: julien.guenole@fau.de (J. Guénolé)
} 
the fabrication of micro- or nano-objects for mechanical testing $[5,6]$. In this context, the damage induced by FIB irradiation has been extensively investigated, both through experiments $[7,8,9,10,11]$ and simulations $[12,13,14]$, as well as in studies combining both these approaches [15]. Most of these studies focus on the influence of ion beam energy, incidence angle and ion type on the damage produced within a specific target material. The measurement of local intrinsic stresses through FIB milling of specific geometries and subsequent determination of the relaxation strain through digital image correlation (DIC) has recently emerged as an innovative application of the FIB [16]. The relation between the visible surface strain relaxation and the intrinsic stress which existed in the material before FIB milling is, however, not straightforward. Among various factors which might interfere with a proper evaluation of stresses, the interplay of intrinsic stresses and irradiation induced damage is largely unknown. Only recently, Pastewka et al. [13] investigated the influence of ion irradiation with grazing-angles on strained samples through atomistic simulations. Our own recent work has shown a clear influence of strain on the damage induced by FIB [17]. Although relatively large strains of the order of $1-4 \%$ were used in these studies, the results are relevant especially for nanostructured materials, which can easily sustain such large strains. For example, Si layers with more than $12 \%$ strain were shown to be present in nanoheterostructures [18]. Consequently, a comprehensive study of the relation between intrinsic strain/stresses and the formation of irradiation induced damage would be of primary importance for assessing the reliability of any stress measurement technique involving FIB milling.

The threshold displacement energy (TDE) and the surface binding energy (SBE) are traditionally used to assess the resistance of a material to irradiation damage. Atomistic simulations are uniquely positioned to determine these properties. Pioneering molecular dynamics calculations of the TDE date back to more than fifty years ago $[19,20]$. The lack of computational power and of sophisticated interatomic potentials, however, confined these studies to consider pair interactions only, leading to plausible albeit imprecise values. With the development of the embedded atom method (EAM) in the 1980s [21], the simulation of many-body interactions became accessible which led to more reliable 
modeling of displacement cascades [22]. Nowadays, calculations of TDE with even more accurate, DFT-informed interatomic potentials [23, 24] and ab-initio calculations [25] pursue the comprehensive understanding of the formation of irradiation damage. A similar historical trend is observed for the calculation of the SBE. Early atomistic studies in the 1970s using two-body potentials [26, 27, 28] were improved by the consideration of many-body interactions [29, 30, 31]. We note here that the calculation of TDE and SBE via atomistic simulations has traditionally been carried out on stress free crystalline structures close to the equilibrium state; the influence of intrinsic strain, to the best of your knowledge, has not yet been considered.

In this work, we address the influence of strain on irradiation damage with a detailed atomistic study of fundamental material properties characterizing the propensity of ion irradiation to induce damage in a material. We present for the first time the computation of the TDE as function of strain using silicon and aluminum as model materials for semiconductors and metals. Furthermore, we compute the surface binding energy (SBE) as function of strain using a statistical approach for amorphized surfaces. These properties are finally correlated with the results of full collision cascade simulations of strained materials.

\section{Methods}

\subsection{Atomistic simulations}

A key aspect of atomistic simulations is the modeling of interatomic interactions. In the current work, we use recent semi-empirical potentials which are fitted to data from density functional theory (DFT) calculations to model the interaction in pure silicon and pure aluminum. The $\mathrm{Si}-\mathrm{Si}$ interactions are modeled with the three-body potential from Stillinger and Weber [32] (denoted in the followoing by $S W$ ), which has been further optimized by Pizzagalli et al. [33] to better reproduce the mechanical properties as well as the TDE of silicon. The $\mathrm{Al}-\mathrm{Al}$ interactions are modeled by the embedded atom method (EAM) potential of Pun and Mishin [34], developed for Ni-Al compounds (denoted Pun in the following). For the $\mathrm{Ga}-[\mathrm{Si}, \mathrm{Al}]$ interaction in ion irradiation, the Ziegler-Biersack-Littmark $(Z B L)$ potential is used [35]. This universal repulsive potential is designed to represent high energy atom collisions, albeit 
ignoring atomic bonding [13, 35].

During a collision cascade, some atoms, in particular the primary knock-on atoms (PKA), have a very high energy. To represent such high-energy interactions, the $S W$ and Pun potentials are smoothly merged to the $Z B L$ potential for short inter-atomic distances following the procedure outlined in Ref. [36, 37]. The merging is made for distances between $1.7-2.0 \AA$ and $1.8-2.1 \AA$ for $\mathrm{Si}$ and $\mathrm{Al}$, respectively. For details of the joining procedure and the influence of the cutoff, see Nordlund et al. [23]. These authors acknowledge the subjective approach commonly used in the literature and recommended the use of simulations based on the density functional theory to highlight the choice. Such ab-initio simulations being out of the scope of the present study, the potential used in the current work is merged based on available literature data on $\mathrm{Si}[36,37]$. Since no previous studies are available for $\mathrm{Al}$, the merging region is defined proportional to the one for $\mathrm{Si}$, based on the nearest neighbor distance in the $\mathrm{Al}$ fcc structure.

The present atomistic simulations are performed with the open-source software LAMMPS (version 7 Dec 2015) [38] running on both central processing unit (CPU) and graphics processing unit (GPU) [39, 40, 41]. Samples are cut from bulk materials and statically relaxed using the conjugate gradient algorithm. The molecular dynamics (MD) simulations are performed with a default timestep of 1.0 fs. Strain is introduced by homogeneously rescaling the coordinates of the atoms and the simulation box in the periodic directions. For bi-axial and uni-axial applied strain, the other directions are kept stress free, allowing for the corresponding Poisson contraction. This models typical experimental situations, e.g. in strained semiconductor devices [42]. More details on the specific MD simulations are given below.

Atomistic configurations are post-processed and visualized using Ovito [43]. The analysis of irradiation induced damage is performed by using the diamond structure identification method [44] and the common neighbour analysis (CNA) method [45] for silicon and aluminum, respectively.

\subsection{Threshold displacement energy}

The TDE is an intrinsic property of bulk materials. It corresponds to the energy required to move an atom from its original stable position to a meta-stable 
position, creating a point defect. This threshold is, however, highly anisotropic. In crystals, it depends on the crystallographic direction and on the thermal noise, which can displace the atoms slightly from their ground state positions. The determination of an averaged TDE can, however, be performed using a statistical approach [25].

In our work, we compute the TDE by using the following scheme, similar to Nordlund et al. [23]. A perfect bulk crystal of 8000 atoms is thermalized at given temperatures $(36 \mathrm{~K}$ and $300 \mathrm{~K})$ and zero pressure. A velocity $v^{\theta, \phi}$ corresponding to an energy of $2 \mathrm{eV}$ is attributed to a randomly chosen atom in a random direction characterized in a spherical coordinate system by $(\theta, \phi)$, and a MD simulation is preformed in the NVE ensemble for 6 ps. $v^{\theta, \phi}$ is then increased by steps of $2 \mathrm{eV}$ till the detection of a defect, at an energy $E^{\mathrm{TDE}}(\theta, \phi)$. Note that only the formation of Frenkel pairs (as detected by the Voronoi cell analysis $[46,45])$ is considered here. The averaged TDE value is constructed over $N=1000$ randomly chosen directions:

$$
\langle\mathrm{TDE}\rangle=\left(\sum_{\psi=0}^{2 \pi} \sum_{\phi=0}^{\pi} E^{\mathrm{TDE}}(\psi, \phi)\right) \times N^{-1} .
$$

\subsection{Surface binding energy}

The SBE is a property related to the surfaces of materials, which characterizes the binding strength of surface atoms. Commonly likened to the sublimation energy [47], it is possible and more accurate to determine this quantity from atomistic simulations $[48,31]$. In this work, we compute the SBE in a scheme similar to Yang et al. [31]. Furthermore, we consider a statistical approach to evaluate the SBE from an amorphous surface, as described below.

Considering the free surface of a perfect crystal at $0 \mathrm{~K}$, a velocity normal to this surface is prescribed to an atom, and MD simulation is performed in the NVE ensemble for 2 ps. Being initially large enough to tear off the atom from the surface, this velocity is decreased step-wise until the atom remains attached to the surface. The energy corresponding to the step right before this last stage is then defined as the SBE.

Note that the system being at $0 \mathrm{~K}$ without any thermal noise, all the atoms belonging to as-cut $\left\{\begin{array}{lll}0 & 0 & 1\end{array}\right\}$ surfaces in FCC and diamond structures are equiva- 
lent. Therefore, no statistical approach is required in this case. Due to covalent bonding, silicon surfaces undergo local reconstruction, as described in the Results section. This reconstruction is obtained by relaxing a pre-disturbed $\left(\begin{array}{lll}0 & 0 & 1\end{array}\right)$ silicon surface.

We extend this approach by using an amorphous surface instead of a perfectly crystalline one to better model the situation during FIB milling. The amorphous structure is obtained by the "random method" described elsewhere [49]. In short, the method consists of filling up a given volume with randomly distributed atoms, by following the density of the amorphous phase. The system is then statically relaxed, annealed at high temperature and statically relaxed again. The resulting silicon amorphous structure has been shown to be in good agreement with the one obtained by the means of other methods [49]. In this case, the atoms belonging to the surface are, however, not well defined. We separate surface atoms from bulk atoms by computing the Voronoi volume of each atoms in the system, and define surface atoms as those atoms with a volume above a specific threshold. A good threshold volume was found to be $30 \AA^{3}$. The SBE for each of these surface atoms is then calculated as described above. The SBE for such an amorphous surface is then obtained by averaging the SBE of individual atoms constituting the surface.

\subsection{Collision cascades}

The irradiation of strained materials is studied by atomistic simulations of full collision cascades. Samples are cut from the bulk and homogeneously scaled to fit the lattice parameter at $300 \mathrm{~K}$ and thermalized at $300 \mathrm{~K}$ by using successively the NVE and NVT ensembles for a total time of 20 ps. Samples are then uniaxially strained and successive cascade simulations are finally run using this initial structure.

During a collision cascade, the heat spike regime requires sufficiently strong energy dissipation while not perturbing the dynamics of the high velocity particles. The kinetic energy provided by the fired ions is therefore dissipated by using a thermostatted region of $2 \mathrm{~nm}$ thickness, parallel to the ion beam and as far as possible from the initial position of the fired ion. Consequently, the position of the thermostatted layer must be adapted for each collision cascade. In this layer, the temperature is controlled by a Berendsen thermostat [50]. 
The thermostatted region acts as an barrier for ion propagation [36], but the influence on the damage formation is negligible and a similar scheme has been previously used successfully [36, 17].

The positions of the $\mathrm{Ga}^{+}$ion are randomly chosen at a distance of $2 \mathrm{~nm}$ from the $(001)$ surface by following a normal distribution with a standard deviation of $2 \mathrm{~nm}$ typical for atomistic simulations [51, 36, 52]. The velocity corresponding to a kinetic energy of $5 \mathrm{keV}$ and in the direction of the beam is attributed to the ion. A MD simulation of the collision cascade is then performed for a maximum time of 15 ps with a variable timestep to ensure that no atoms would be displaced by a distance larger than $0.01 \mathrm{~nm}$ during one MD step. Because of the high energy involved, sputtering can occur. An atom is considered as sputtered and then removed from the simulation if it leaves the simulation box, i.e. the distance between the atom and the closest surface is larger than two times the cutoff of the potential. For all ions, the collision cascade is finished by the end of these $15 \mathrm{ps}$, with a final global temperature below $400 \mathrm{~K}$. A final MD simulation is performed for 15 ps to slowly cool down the system from the current temperature to $300 \mathrm{~K}$, so as to get the structure ready for the next collision cascade.

\section{Results}

\subsection{Threshold displacement energy}

The TDE is calculated at a given uniaxial applied strain in the [010] direction for 1000 randomly chosen spatial directions. The average and the standard deviation of this average, i.e. standard error, are calculated over the entire population, leading to one data point per strain value.

Fig. 1 shows the TDE at $36 \mathrm{~K}$ and $300 \mathrm{~K}$. Temperatures of $36 \mathrm{~K}$ and lower are commonly used in atomistic simulations $[23,25]$ and therefore included in the present work. Fig. 1 shows a global increase of the TDE from $36 \mathrm{~K}$ to simulations at $300 \mathrm{~K}$, however, the trends with respect to strain remain unchanged.

The TDE is usually determined using unmodified potentials [23, 24]. For the simulations of collision cascades, however, modified potentials are used in which the the ZBL potential is merged to the material specific potential for short interaction distances to improve the description of high energy collisions [53, 54]. 


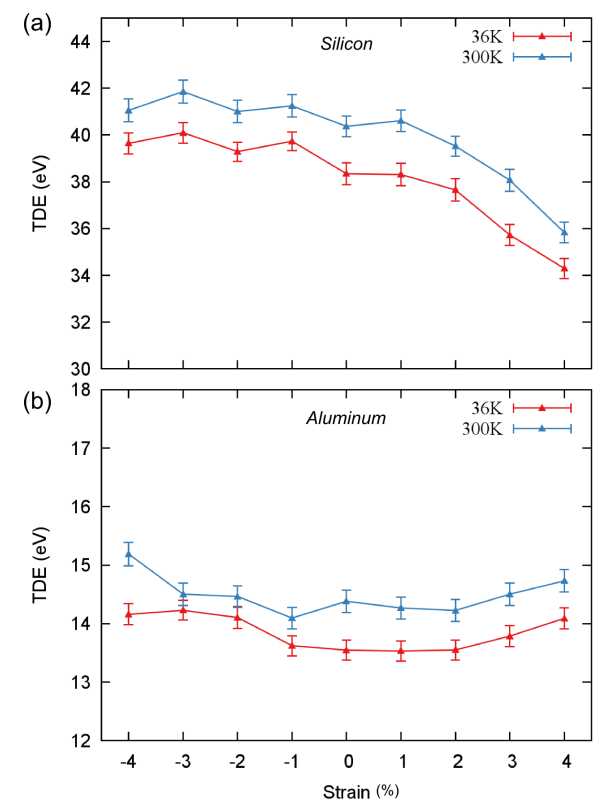

Figure 1: Average threshold displacement energy as function of uniaxial applied strain along the [010] direction at $36 \mathrm{~K}$ and $300 \mathrm{~K}$, for (a) Si and (b) Al. Tensile applied strains are taken as positive, compressive applied strains as negative. Error bars correspond to the standard error of the data set (1000 independent values each). Solid lines serve as guides to the eye.

It is therefore important to determine the TDE also for the modified potential.

Fig. 2 shows the TDE at $36 \mathrm{~K}$ as a function of uniaxial applied strain along the [010] direction for the original potentials (red) and the potentials modified by merging the ZBL potential (green). We note that the data corresponding to the material specific multi-body potentials is identical to that presented in Fig. 1 at $36 \mathrm{~K}$. Further details on the distribution of TDE values used for averaging are presented as box-and-whisker plots in Fig. 2a,b. Such box-and-whisker plots represent the two central quartiles in a box, the central line being the median. The highest and lowest quartiles are represented by the whiskers.

To emphasize the influence of the merging of the $Z B L$ to the material specific potentials, the cohesive energies of perfect Si (Fig. 2c) and Al (Fig. 2d) crystals are calculated as a function of hydrostatic strain, which is applied by homogeneously scaling the coordinates of the fully periodic atomistic structure. Here, the strain is represented as $n n / n n_{0}$ with $n n$ being the nearest neighbor distance and $n n_{0}$ being the nearest neighbor distance of the crystalline ground state.

The TDE as function of applied strain for certain specific directions is shown 

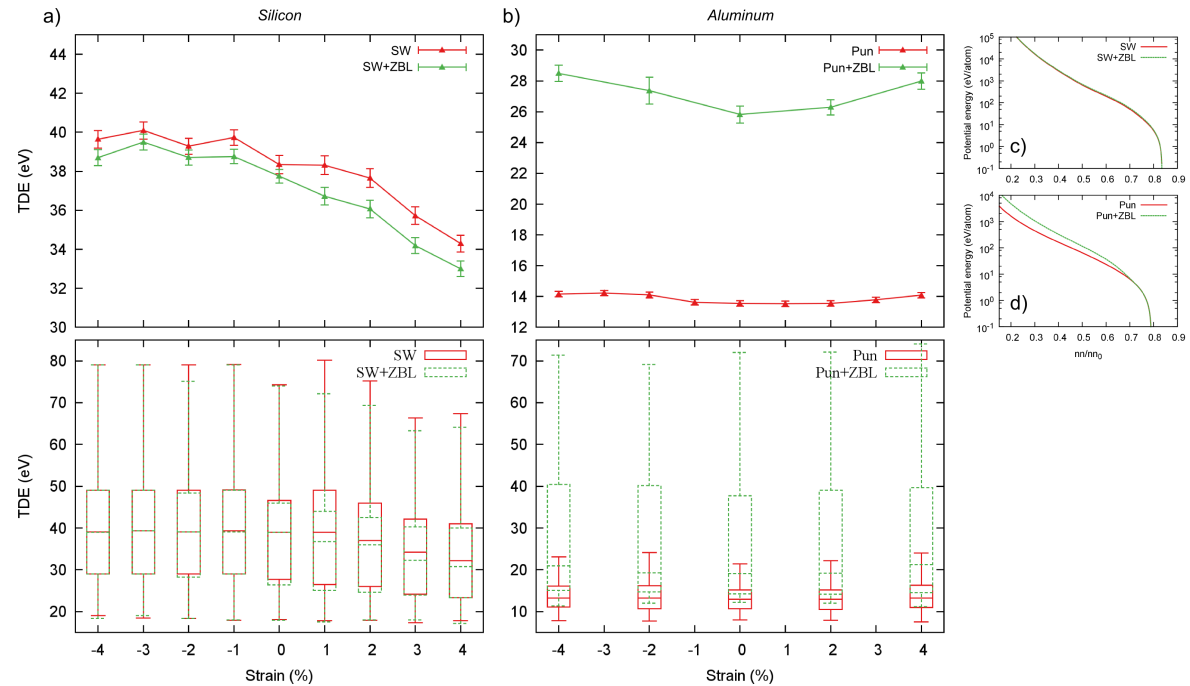

Figure 2: Influence of modifying the potential by merging it with the $Z B L$ potential on the threshold displacement energy (TDE) as function of applied strain in [010] direction for (a) $\mathrm{Si}$ and (b) Al. The upper plots show the averaged TDE and the corresponding standard error of the data set (1000 independent values each). Solid lines are guides to the eye. The box-andwhisker plots show the distribution of these $1000 \mathrm{TDE}$ values, the inner box line representing the median of each data set. (c) and (d) show the cohesive energies of perfectly crystalline Si and $\mathrm{Al}$, respectively, as a function of hydrostatic strain.

for $36 \mathrm{~K}$ in Fig. 3. The red values are identical to those shown on Fig. 1-2 and encompass all spatial directions. The orange (brown) values encompass the TDE in the directions normal (parallel) to the applied strain directions [0 10$]$. Samples are globally stress-free and undergo Poisson contraction. The strain state of directions normal to the tensile (compressive) applied strain are under compression (tension). Note that not only the exact direction normal or parallel to the imposed strain, but all the directions included in a solid angle of $60^{\circ}$ are considered for this purpose. In these cases, the TDE was averaged over 200-300 values, less than the 1000 values used for the all directions averaged TDE. Hence, the standard error is slightly increased, emphasizing the weaker statistics.

Fig. 4 shows the TDE at $36 \mathrm{~K}$ as function of applied strain for different applied strain modes: uniaxial (red), equibiaxial (blue) and hydrostatic (green). As before, the directions not strained are stress-free. The resulting strain states of the system are thus more complex; samples with compressive (tensile) uniaxial/biaxial applied strain exhibit tensile (compressive) strain in the perpendicular directions. 


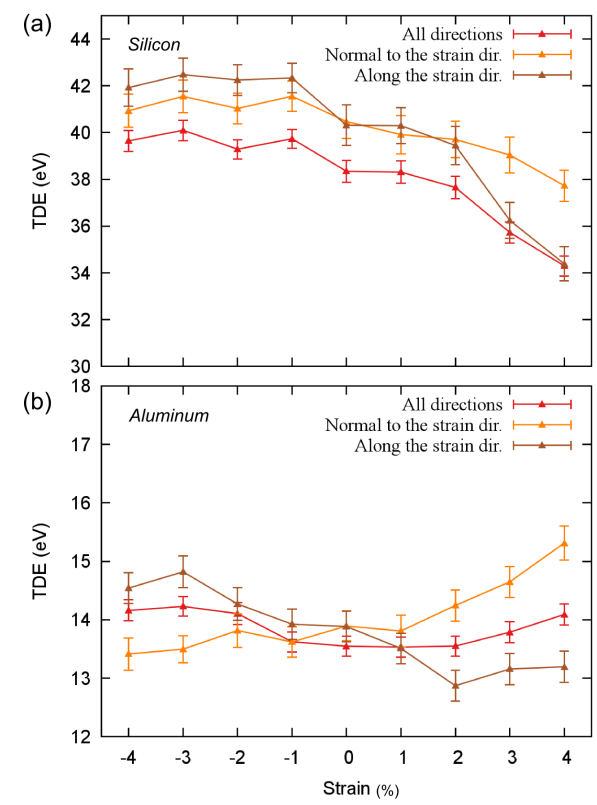

Figure 3: Average threshold displacement energy as function of the spatial directions considered, for (a) Si and (b) Al. All directions are considered for the red points. Only the directions in a solid angle of $60^{\circ}$ normal (parallel) to the applied strain direction [010] are considered for the orange (brown) points. Error bars correspond to the standard error of the data set. Solid lines serve as guides to the eye.

\subsection{Surface binding energy}

Fig. 5 shows the SBE as function of uniaxial applied strain calculated for different situations. As a reference, the experimental values of sublimation energy are also provided [55], see also the Supplementary Materials [56]. Direct computations or measurements of the SBE are rarely found in the literature.

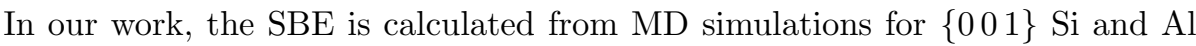
surfaces (Fig. 5). Unlike metals, covalent materials like silicon undergo short range surface reconstruction. For the $\left\langle\begin{array}{lll}0 & 0 & 1\end{array}\right\rangle$ surfaces considered here, the reconstruction follows the $\pi$-bonded chain model of Pandey [57], named $p(2 \times 1)$ for symmetry reasons, and characterized by the formation of bonds between surface atoms in the $\langle 011\rangle$ directions. Instead of being two times coordinated, the reconstructed surface atoms are three times coordinated and thus more difficult to sputter. The SBE for as-cut and reconstructed Si surfaces are consequently different as can be seen in Fig. 5a.

As described in the methods section, we also calculate an average SBE for an amorphous surface. The data points shown with black triangles in Fig. 5 


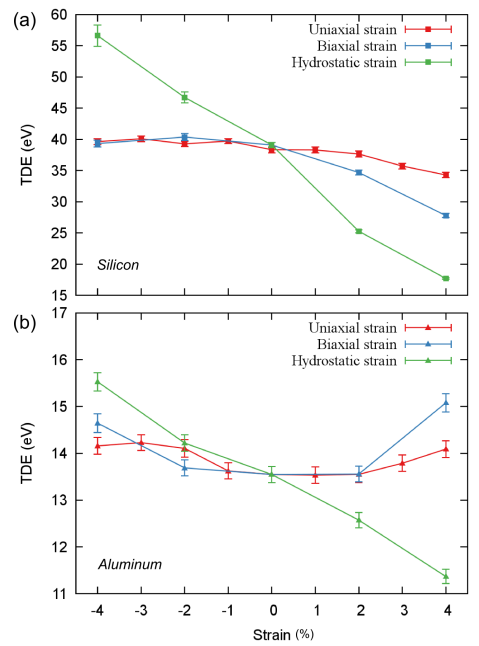

Figure 4: Average threshold displacement energy as function of applied strain mode, for (a) $\mathrm{Si}$ and (b) Al. Applied strain modes considered are uniaxial ([0 10$]$, red), equibiaxial (along $\left[\begin{array}{lll}0 & 1 & 0\end{array}\right]$ and $\left[\begin{array}{lll}1 & 0 & 0\end{array}\right]$ blue) and hydrostatic (green). Error bars correspond to the standard error of the data set (1000 independent values each). Solid lines are guides to the eye.

along with the standard deviation, show a clear increase of the energy required to sputter an atom from an amorphous $\mathrm{Si}$ surface, in comparison to as-cut surfaces. The SBE of an amorphous $\mathrm{Al}$ surface appears also slightly larger than for an as-cut surface. As indicated by the standard deviation, however, significant data spread is observed.

A table summarizing SBE data is provided in the Supplementary Materials $[56]$.

\subsection{Irradiation cascades}

In order to study the effect of the aforementioned influence of strain on TDE and SBE on irradiation damage, atomistic simulations of ion irradiation were performed on strained silicon samples. The evolution of irradiation induced damage throughout the sample is shown in Fig 6. Fig. 6(a) shows perspective views of the entire sample after a certain number of fired ions (corresponding to the number of simulated collision cascades). Fig. 6(b) shows projected views of a $4 \mathrm{~nm}$ thick slice, extracted from the region with the highest incoming ion density. Fig. 6(c) shows perspective views similar to the first row, excluding the atoms in perfect crystalline environment, and thus providing a better overview of the stable damage produced by the ion irradiation. 


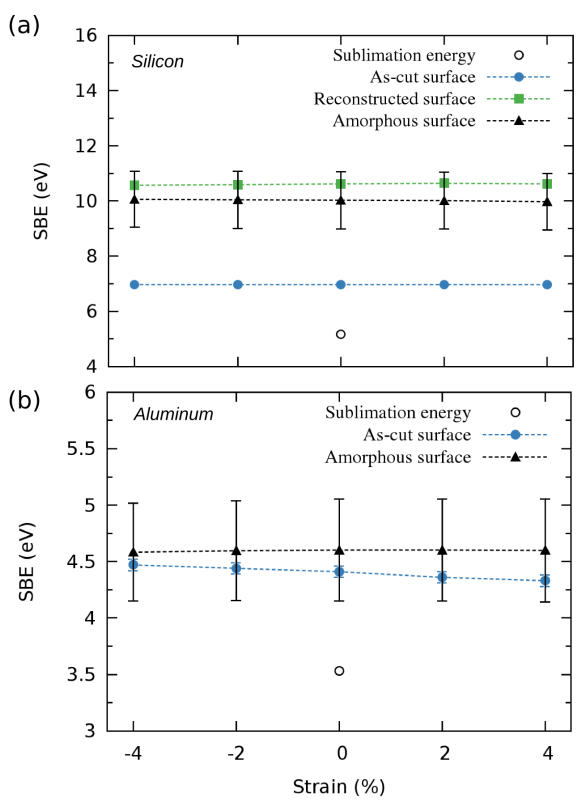

Figure 5: Surface binding energy for (a) a (0 01 ) silicon surface and (b) a (0 01$)$ aluminum surface calculated at different uniaxial tensile applied strains. Empty black circles represent the experimentally determined sublimations energies [55]. Full blue circles: surface binding energy for as-cut surface; full green squares: surface binding energy for reconstructed surface (silicon only); full black triangles: surface binding energy for disordered surface with random roughness. Error bars correspond to the sum of standard deviation of the data set and the systematic error, and describe the spread of the SBE values. Dashed lines are guides to the eye.

Only stable damage configurations are considered here. During a typical collision cascade, the area where the collisions occur is in the so-called heat spike regime. The very high temperature reached at the atomic scale melts the sample locally resulting in significant amount of damage. However, with almost all excess thermal energy being dissipated before the occurrence of the next collision cascade, the melted region undergoes a dramatic recrystallization. The remaining damage is then extremely stable at the temperature considered in this study.

The amount of damage can be obtained by evaluating the structural environment of each atom. The atoms are classified as perfectly crystalline (diamond cubic or fcc), or as defect atoms. Note that this essentially results in surface atoms being classified as defect atoms, as seen in Fig. 6(c). For irradiation up to some tens of ions, damage is primarily localized in the region around the path of the ion. With 50 ions, the damaged area is significantly larger and a clear amorphous zone can be observed. Further increase in ion dosage increases 


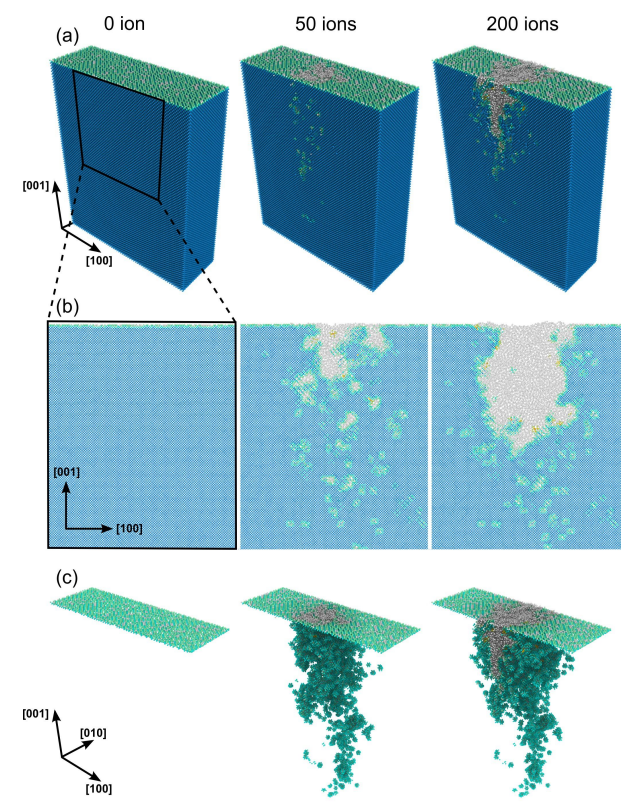

Figure 6: Induced damage in collision cascade simulations of $5 \mathrm{keV} \mathrm{Ga}^{+}$ions in $\mathrm{Si}$ at $300 \mathrm{~K}$. (a) perspective view and (b) projected view of a slice with a thickness of $4 \mathrm{~nm}$ located at the simulation box center, i.e. the highest concentration of incoming ions. (c) perspective view where atoms in a perfect cubic diamond structure environment (until the second neighbor) are hidden to highlight irradiation induced damage. Atoms colored according the diamond structure identification method implemented in OviTo (blue - cubic diamond, cyan/green cubic diamond 1st/2nd neighbor, white - non-diamond structure).

the damaged zone dramatically. A large fully amorphous region is formed, surrounded by smaller disordered regions.

Counting atoms in a perfect crystallographic environment and those classified as defect allows the quantification of the damage induced by the irradiation. Tab. 1 presents the amount of atoms classified as perfect and defect, along the sputtered atoms. The ratio over the entire sample of atoms classified as defect because of ion irradiation is presented in the last row. Before irradiation, atoms belonging to the free surface are also classified as defects; the number of defect atoms due to irradiation is zero. Without applied strain, the irradiated sample exhibits a fraction of $11.2 \%$ of defects due to irradiation. A similar fraction is also observed for samples irradiated under compressive applied strains. By contrast, the fraction of defect atoms due to irradiation shows a significant increase for tensile applied strains. Quantifying sputtered atoms reveals slight variations with strain. With both compressive and tensile applied strain, the sputter yield is increased by approximately $1 \%$. For a compressive applied strain of $-4 \%$, 


\begin{tabular}{r|r|rrrrr}
\hline Irradiated & no & \multicolumn{5}{c}{ yes } \\
\hline Strain (\%) & - & -4 & -2 & 0 & +2 & +4 \\
\hline Perfect atoms (\#) & 699,926 & 621,710 & 620,390 & 620,342 & 613,335 & 608,837 \\
Sputtered atoms (\#) & 0 & 2,350 & 348 & 308 & 366 & 369 \\
Defect atoms (\#) & 12,874 & 88,740 & 92,062 & 92,150 & 99,099 & 103,594 \\
Irradiation defect atoms (\%) & 0.0 & 11.0 & 11.2 & 11.2 & 12.2 & 12.8 \\
\hline
\end{tabular}

Table 1: Fraction of atoms belonging to irradiation induced damage as function of uniaxial applied strain along the [010] direction. Atoms in perfect cubic diamond structure environment are counted as Perfect atoms. Atoms removed by sputtering are counted as Sputtered atoms. Atoms not in perfect cubic diamond structure environment are counted as Defect atoms. Note that the sample before irradiation (first column) shows Defect atoms because of the free surface. The ratio of atoms corresponding to irradiation induced damage, named Irradiation defect atoms, is the ratio over the entire sample of the sum of Defect atoms and Sputtered atoms, reduced by removing surface atoms (e.g. Defect atoms before irradiation).

the sample undergoes local surface boiling, which leads to brutal sputtering for some incident ions only. The final amount of sputtered atoms is thus larger than for the other simulations. Such stochastic effects are hard to prevent in such short time-scale simulations, but don't contradict the other results.

\section{Discussion}

Our results show a clear influence of the strain state on the TDE. The various aspects which could contribute to this influence are discussed in the following.

\subsection{Influence of interatomic potential}

While performing atomistic simulations of ion irradiation, it is commonly accepted practice to add a strong repulsive part to the interatomic potential describing the target material, see sec. 2.1. The details of the merging of these two potentials $-S W / Z B L$ and $P u n / Z B L$ for Si and Al, respectively - are known to be subjective rather than based on physical principles [23] and to strongly influence the resulting interatomic interactions. According to our results, joining the $Z B L$ to the $S W$ potential doesn't change the strain dependency but lowers the TDE (Fig. 2a). This decrease of the TDE in Si is, however, negligible and is smaller than the decrease of the TDE due to temperature variation (Fig. 1a). Surprisingly, our results show a completely different effect for Al (Fig. 2b); joining the $Z B L$ to the Pun potential doesn't change the trend of strain dependency, but strongly increases the TDE values. The TDE values computed with $Z B L$ potential are twice the values of TDE without $Z B L$. 
An increase of the averaged TDE of $\mathrm{Si}$ and $\mathrm{Al}$ can have different origins. Both, an increased spread favoring higher values in the individual calculations, or a global shift of the TDE, might explain such increase of the averaged TDE. Looking at the distribution of the TDE over all spatial directions (Fig. 2a,b with boxplots) shows that the TDE distributions for $S W$ and $S W / Z B L$ mostly overlap, with a similar trend independent of the presence of the $Z B L$ potential.

Conversely, the TDE distribution is dramatically widened from Pun to $P u n / Z B L$, with the two central quartiles being 5 times larger in the Pun/ZBL case. The lowest values of the distribution are also strongly increased, being close the the median of the TDE for Pun. Thus, joining the $Z B L$ to the Pun potential not only increases the averaged TDE of $\mathrm{Al}$, but prevents low TDE values while strongly favoring TDE values higher than the most probable TDE for the Pun potential alone.

The influence of adding the $Z B L$ potential to the material specific potential can be assessed by calculating the cohesive energy as function of hydrostatic strain, see Figs. 2c and d. Compared to the three-body potential for Si, the EAM potential for $\mathrm{Al}$ shows a significant increase of the cohesive energy for larger compressive applied strains through the addition of the $Z B L$ potential. The difference can be explained by the fact that the description of the covalent bonding in the $S W$ potential does not take into account the environment of the bonds, whereas the EAM potential is environment-dependent. Consequently, any modification of the EAM potential should in principle not only involve the pair-part of the potential, but also the environment-dependent terms. However, this is not usually considered [23].

The present results suggest that realistically modeling the formation of ion irradiation damage and sputtering in metals requires a more sophisticated treatment of the short-range interactions, and highlight the importance of using identical potentials when interpreting collision cascades in terms of the TDE.

\subsection{Influence of strain state}

Although merging the potentials with the $Z B L$ potential does influence the magnitude of the TDE, it does not affect its behavior with respect to uniaxial applied strain. The cohesively bonded semiconductor $(\mathrm{Si})$ shows a clear decrease of the TDE with uniaxial tensile applied strain, whereas for the metallic bonded 
aluminum an opposite trend can be deduced, see Fig. 1. In general, the situation is, however, more complex, as highlighted in Fig. 3 and 4: the influence of applied strain on the TDE depends on the spatial direction for which the TDE is determined and the applied strain mode. By looking at hydrostatically strained samples, it is clear that tensile strains decrease the TDE, whereas compressive strains increase the TDE, in both $\mathrm{Al}$ and $\mathrm{Si}$, see Fig. 4. This observation is a direct consequence of the anharmonicity of the cohesive energy: the cohesive energy shows a strong increase in hydrostatic compression and a smaller increase in tension [58]. The process of creating a Frenkel-pair should thus be influenced to a different degree in tension than in compression. For more complex strain states, which involve both, shortened and elongated bonds and subsequently changed bond angles, like in the case of uni- and biaxial applied strains, the situation is less clear. In these cases, $\mathrm{Si}$ and $\mathrm{Al}$ show contradictory trends, which could be attributed to the stronger angular dependency of the bonding in Si compared to Al.

\subsection{Influence of surface morphology}

The SBEs calculated in this study are significantly higher than the usually considered values, e.g. the sublimation energy [55] or the ones calculated by analytical models [47]. Values for the SBE that are 10-35\% larger than the sublimation energy have already been put forward in the pioneering work of Jackson [26], who also included the effect of surface relaxation. Similary SBE values were also reported in later studies, highlighting effects of slow collisions processes [48] or many-body interactions [29]. Atomistic simulations take into account the local atomic relaxation around the vacancy left behind by the sputtered atom, and in doing so provide accurate values for the SBE [31, 59]. For MD simulations, the SBE is expected to be up to $40 \%$ larger than the sublimation energy [59], see also the recent results on MD simulations on tungsten [31]. These observations are confirmed by our calculations on aluminium. The SBE for the as-cut $\mathrm{Al}$ surface is approximately $30 \%$ larger than the sublimation energy.

For covalent materials with directional bonding, the effect of local atomic relaxation can be much stronger than for metals [59], as observed with the sputtering from silicon surfaces $[60,61]$. Our calculations on silicon exhibit a 
similar trend. The SBE for the as-cut $\{001\}$ Si surface is approximately $50 \%$ larger than the sublimation energy. For the reconstructed $\{001\}$ Si surface, the difference is even more pronounced, with a SBE 130\% larger than the sublimation energy. A detailed analysis of the atomic mechanisms which take place in the simulations to determine the SBE, reveal crucial aspects which explain the dramatic increase of the values from a as-cut to a reconstructed surface: as an atom is torn off from the surface, its direct neighbors undergo reorganization to lower their energies. The reorganization is more pronounced for the reconstructed surface than for the as-cut surface; in the $p(2 \times 1)$ surface, atoms are 3 -fold coordinated, compared to the 2 -fold coordinated atoms of the the as-cut $\{001\}$ Si surface.

It is known from literature that sputtering an atom from a surface is a highly anisotropic process, depending on the angle of ejection [27] with respect to the surface. In addition, an anisotropy due to the direction of the sputtered atom with respect to the local crystallography of the surface could be expected for crystalline surfaces. For amorphous surfaces, no anisotropy with respect to local crystallography exists. However, the amorphous surfaces show a certain roughness. The SBE therefore will have different values depending on the local angle of ejection. The averaged SBE is, nevertheless, of the order of the SBE determined for the as-cut and reconstructed surfaces for $\mathrm{Al}$ and $\mathrm{Si}$, respectively, see Fig. 5. This relation between the SBE of undamaged and amorphous silicon surfaces is directly related to the coordination number of surface atoms, as mentioned by Gades et al. [30]; atoms of an as-cut Si surface are 2 times coordinated, whereas atoms belonging to the reconstructed and amorphous $\mathrm{Si}$ surfaces in our simulations are 3 times coordinated. As pointed out in the methods section, the surface roughness of amorphous surfaces requires a statistical approach. As a result, the average SBE value shows a large standard deviation, see Fig. 5. Interestingly, the scatter of $2 \mathrm{eV}$ is similar for both model materials used in the current work, emphasizing its dependence on roughness rather than on the material.

The significant increase of the SBE observed for Si has important consequences for the modeling of ion milling processes, as the ions will mostly impinge on an already amorphized surface, for which the widely used estimates of the 
SBE derived from perfect surfaces or from sublimation energies are inadequate.

\subsection{Collision cascades and TDE}

The atomistic simulations of ion irradiation using strained silicon corroborate the observed trends in the strain dependence of the TDE. The uniaxially strained samples clearly show increased damage formation for tensile strains, see Tab. 1 as predicted by the strain-dependent TDE. This highlights the importance of taking into account the strain state of the milled material for more accurate modeling of FIB milling. In this context, the strain-dependent TDE becomes a material parameter of fundamental importance.

\section{Conclusions}

Atomistic simulations have been performed on silicon and aluminum to compute the averaged threshold displacement energy (TDE) and surface binding energy (SBE) of $\left\{\begin{array}{lll}0 & 0\end{array}\right\}$ surfaces for different strain states. In addition, full collision cascades have been simulated for uniaxially strained silicon. Our results suggest that realistically modeling ion irradiation damage in metals requires a more sophisticated treatment of the short-range interactions compared to covalently bonded semiconductors. For both, $\mathrm{Si}$ and $\mathrm{Al}$, hydrostatic tensile strains decrease the TDE whereas compressive strains increase the TDE, which is expected from the anharmonic nature of the cohesive energy. For more complex strain states, like in the case of uni- and biaxial applied strains, which involve both, shortened and elongated bonds, the different dependence of the bonding energy on bond angles in metals and semiconductors leads to qualitative differences in the strain dependence of the TDE. Cascade simulations on uniaxially strained silicon confirm this observation and underline the importance of the strain-dependent TDE as a fundamental material parameter. The SBE for realistic (reconstructed or amorphized) surfaces is shown to be significantly larger than the sublimation energy, which is often used as approximation of the SBE. Consequently, models of FIB-milling could be improved by taking into account the strain dependence of the TDE, and SBEs derived from more realistic surfaces. 


\section{Acknowledgments}

This work was supported by the European Union, within the large collaborative project ISTRESS, Grant Agreement No. 604646. EB acknowledges the support of the Cluster of Excellence Engineering of Advanced Materials (EAM) and the Central Institute of Scientific Computing (ZISC). JG would like to thank J. Amodeo, Lyon, France and J. Godet, Poitiers, France for fruitful discussions.

\section{References}

[1] A. A. Tseng, Recent developments in micromilling using focused ion beam technology, Journal of Micromechanics and Microengineering 14 (2004) $\mathrm{R} 15$.

[2] C. A. Volkert, A. M. Minor, Focused ion beam microscopy and micromachining, MRS Bulletin 32 (2007) 389-399.

[3] H.-J. Kang, J. Kim, J. Oh, T. Back, H. Kim, Ultra-thin TEM sample preparation with advanced backside FIB milling method, Microscopy and Microanalysis 16 (2010) 170-171.

[4] D. Larson, D. Foord, A. Petford-Long, H. Liew, M. Blamire, A. Cerezo, G. Smith, Field-ion specimen preparation using focused ion-beam milling, Ultramicroscopy 79 (1999) $287-293$.

[5] D. Kiener, W. Grosinger, G. Dehm, R. Pippan, A further step towards an understanding of size-dependent crystal plasticity: In situ tension experiments of miniaturized single-crystal copper samples, Acta Materialia 56 (2008) $580-592$.

[6] D. Kiener, C. Motz, G. Dehm, Micro-compression testing: A critical discussion of experimental constraints, Materials Science and Engineering: A 505 (2009) $79-87$.

[7] D. Kiener, C. Motz, M. Rester, M. Jenko, G. Dehm, FIB damage of cu and possible consequences for miniaturized mechanical tests, Materials Science and Engineering: A 459 (2007) $262-272$. 
[8] A. Lugstein, W. Brezna, G. Hobler, E. Bertagnolli, Method to characterize the three-dimensional distribution of focused ion beam induced damage in silicon after $50 \mathrm{kev}$ ga+ irradiation, Journal of Vacuum Science \& Technology A 21 (2003) 1644-1648.

[9] S. Rubanov, P. R. Munroe, FIB-induced damage in silicon, Journal of Microscopy 214 (2004) 213-221.

[10] D. A. Cullen, D. J. Smith, Assessment of surface damage and sidewall implantation in algan-based high electron mobility transistor devices caused during focused-ion-beam milling, Journal of Applied Physics 104 (2008) -.

[11] Z. Wang, T. Kato, T. Hirayama, N. Kato, K. Sasaki, H. Saka, Surface damage induced by focused-ion-beam milling in a si/si pn junction crosssectional specimen, Applied Surface Science 241 (2005) 80 - 86. The 9th International Symposium on Advanced Physical Fields.

[12] Z. Tong, Z. Xu, W. Wu, X. Luo, Molecular dynamic simulation of lowenergy FIB irradiation induced damage in diamond, Nuclear Instruments and Methods in Physics Research Section B: Beam Interactions with Materials and Atoms 358 (2015) 38 - 44.

[13] L. Pastewka, R. Salzer, A. Graff, F. Altmann, M. Moseler, Surface amorphization, sputter rate, and intrinsic stresses of silicon during low energy ga + focused-ion beam milling, Nuclear Instruments and Methods in Physics Research Section B: Beam Interactions with Materials and Atoms 267 (2009) $3072-3075$. Proceedings of the Ninth International Conference on Computer Simulation of Radiation Effects in Solids.

[14] Y. Xiao, F. Fang, Z. Xu, X. Hu, Annealing recovery of nanoscale silicon surface damage caused by ga focused ion beam, Applied Surface Science 343 (2015) 5669.

[15] P. D. Lane, G. J. Galloway, R. J. Cole, M. Caffio, R. Schaub, G. J. Ackland, Validating molecular dynamics with direct imaging of radiation damage debris, Phys. Rev. B 85 (2012) 094111. 
[16] A. M. Korsunsky, M. Sebastiani, E. Bemporad, Focused ion beam ring drilling for residual stress evaluation, Materials Letters 63 (2009) 1961 1963.

[17] J. Guénolé, A. Prakash, E. Bitzek, Atomistic simulations of focused ion beam machining of strained silicon (2016). Submitted.

[18] K.-C. Lu, W.-W. Wu, H.-W. Wu, C. M. Tanner, J. P. Chang, L. J. Chen, K. N. Tu, In situ control of atomic-scale si layer with huge strain in the nanoheterostructure nisi/si/nisi through point contact reaction, Nano Letters 7 (2007) 2389-2394.

[19] J. B. Gibson, A. N. Goland, M. Milgram, G. H. Vineyard, Dynamics of radiation damage, Phys. Rev. 120 (1960) 1229-1253.

[20] C. Erginsoy, G. H. Vineyard, A. Englert, Dynamics of radiation damage in a body-centered cubic lattice, Phys. Rev. 133 (1964) A595-A606.

[21] S. M. Foiles, M. I. Baskes, M. S. Daw, Embedded-atom-method functions for the fcc metals cu, ag, au, ni, pd, pt, and their alloys, Phys. Rev. B 33 (1986) 7983-7991.

[22] D. J. Bacon, T. D. de la Rubia, Molecular dynamics computer simulations of displacement cascades in metals, Journal of Nuclear Materials 216 (1994) $275-290$.

[23] K. Nordlund, J. Wallenius, L. Malerba, Molecular dynamics simulations of threshold displacement energies in fe, Nuclear Instruments and Methods in Physics Research Section B: Beam Interactions with Materials and Atoms 246 (2006) $322-332$.

[24] E. Gonzalez, Y. Abreu, C. Cruz, I. Piera, A. Leyva, Molecular-dynamics simulation of threshold displacement energies in batio3, Nuclear Instruments and Methods in Physics Research Section B: Beam Interactions with Materials and Atoms 358 (2015) 142 - 145.

[25] E. Holmström, A. Kuronen, K. Nordlund, Threshold defect production in silicon determined by density functional theory molecular dynamics simulations, Phys. Rev. B 78 (2008) 045202. 
[26] D. P. Jackson, Binding energies in cubic metal surfaces, Radiation Effects 18 (1973) 185-189.

[27] D. P. Jackson, Surface ejection in single crystal sputtering, Canadian Journal of Physics 53 (1975) 1513-1523.

[28] H. H. Andersen, The depth resolution of sputter profiling, Applied physics 18 (1979) 131-140.

[29] H. Gades, H. M. Urbassek, Pair versus many-body potentials in atomic emission processes from a cu surface, Nuclear Instruments and Methods in Physics Research Section B: Beam Interactions with Materials and Atoms 69 (1992) $232-241$.

[30] H. Gades, H. M. Urbassek, Surface binding energies of alloys: a many-body approach, Nuclear Instruments and Methods in Physics Research Section B: Beam Interactions with Materials and Atoms 88 (1994) 218 - 228.

[31] X. Yang, A. Hassanein, Atomic scale calculations of tungsten surface binding energy and beryllium-induced tungsten sputtering, Applied Surface Science 293 (2014) 187 - 190.

[32] F. H. Stillinger, T. A. Weber, Computer simulation of local order in condensed phases of silicon, Physical Review B 31 (1985) 5262-5271.

[33] L. Pizzagalli, J. Godet, J. Guénolé, S. Brochard, E. Holmstrom, K. Nordlund, T. Albaret, A new parametrization of the stillinger-weber potential for an improved description of defects and plasticity of silicon, Journal of Physics: Condensed Matter 25 (2013) 055801.

[34] G. P. Pun, Y. Mishin, Development of an interatomic potential for the ni-al system, Philosophical Magazine 89 (2009) 3245-3267.

[35] J. F. Ziegler, J. P. Biersack, The stopping and range of ions in matter, Springer, 1985.

[36] E. Holmström, J. Kotakoski, L. Lechner, U. Kaiser, K. Nordlund, Atomicscale effects behind structural instabilities in si lamellae during ion beam thinning, AIP Advances 2 (2012) -. 
[37] K. Nordlund, J. Keinonen, A. Kuronen, Effect of the interatomic si-sipotential on vacancy production during ion implantation of si, Physica Scripta 1994 (1994) 34.

[38] S. Plimpton, Fast parallel algorithms for short-range molecular dynamics, Journal of Computational Physics 117 (1995) 1 - 19.

[39] W. M. Brown, P. Wang, S. J. Plimpton, A. N. Tharrington, Implementing molecular dynamics on hybrid high performance computers - short range forces, Comp. Phys. Comm. 182 (2011) 898-911.

[40] W. M. Brown, A. Kohlmeyer, S. J. Plimpton, A. N. Tharrington, Implementing molecular dynamics on hybrid high performance computers particle-particle particle-mesh, Comp. Phys. Comm. 183 (2012) 449-459.

[41] W. M. Brown, Y. Masako, Implementing molecular dynamics on hybrid high performance computers three-body potentials, Comp. Phys. Comm. 184 (2013) 2785-2793.

[42] S. Bedell, A. Khakifirooz, D. Sadana, Strain scaling for cmos, MRS Bulletin 39 (2014) 131-137.

[43] A. Stukowski, Visualization and analysis of atomistic simulation data with ovitothe open visualization tool, Modelling and Simulation in Materials Science and Engineering 18 (2010) 015012.

[44] E. Maras, O. Trushin, A. Stukowski, T. Ala-Nissila, H. Jnsson, Global transition path search for dislocation formation in ge on si(001), Computer Physics Communications 205 (2016) 13-21.

[45] A. Stukowski, Structure identification methods for atomistic simulations of crystalline materials, Modelling and Simulation in Materials Science and Engineering 20 (2012) 045021.

[46] C. H. Rycroft, Voro++: A three-dimensional voronoi cell library in c++, Chaos 19 (2009) 041111.

[47] Y. Kudriavtsev, A. Villegas, A. Godines, R. Asomoza, Calculation of the surface binding energy for ion sputtered particles, Applied Surface Science 239 (2005) $273-278$. 
[48] R. Kelly, The surface binding energy in slow collisional sputtering, Nuclear Instruments and Methods in Physics Research Section B: Beam Interactions with Materials and Atoms 18 (1987) 388 - 398.

[49] J. Guénolé, J. Godet, S. Brochard, Plasticity in crystalline-amorphous coreshell si nanowires controlled by native interface defects, Physical Review B 87 (2013) 045201.

[50] H. J. C. Berendsen, J. P. M. Postma, W. F. van Gunsteren, A. DiNola, J. R. Haak, Molecular dynamics with coupling to an external bath, The Journal of Chemical Physics 81 (1984) 3684-3690.

[51] M. F. Russo, M. Maazouz, L. A. Giannuzzi, C. Chandler, M. Utlaut, B. J. Garrison, Trench formation and lateral damage induced by gallium milling of silicon, Applied Surface Science 255 (2008) 828 - 830.

[52] K. Das, J. B. Freund, H. T. Johnson, Mechanisms of material removal and mass transport in focused ion beam nanopore formation, Journal of Applied Physics 117 (2015) 085304.

[53] K. Nordlund, Molecular dynamics simulation of ion ranges in the $1100 \mathrm{kev}$ energy range, Computational Materials Science 3 (1995) 448 - 456.

[54] K. Nordlund, M. Ghaly, R. S. Averback, M. Caturla, T. Diaz de la Rubia, J. Tarus, Defect production in collision cascades in elemental semiconductors and fcc metals, Phys. Rev. B 57 (1998) 7556-7570.

[55] J. M. W. Chase, NIST-JANAF Thermochemical Tables Set, SPRINGER VERLAG GMBH, 1998.

[56] Supplementary materials, ????

[57] K. C. Pandey, New $\pi$-bonded chain model for si(111)- $(2 \times 1)$ surface, Physical Review Letters 47 (1981) 1913-1917.

[58] D. G. Pettifor, Bonding and structure of molecules and solids, Oxford University Press, USA, 1995.

[59] R. Behrisch, W. Eckstein, Sputtering by Particle Bombardment: Experiments and Computer Calculations from Threshold to MeV Energies, volume 110, Springer, 2007. 
[60] H. Feil, H. J. W. Zandvliet, M.-H. Tsai, J. D. Dow, I. S. T. Tsong, Random and ordered defects on ion-bombarded si(100)-(2x1) surfaces, Phys. Rev. Lett. 69 (1992) 3076-3079.

[61] V. Zinovyev, L. Aleksandrov, A. Dvurechenskii, K.-H. Heinig, D. Stock, Papers presented at the european materials research society 1993 spring conference, symposium c: Ion beam, plasma, laser and thermally-stimulated deposition processes, strasbourg, france, may 47, 1993 modelling of layerby-layer sputtering of si(111) surfaces under irradiation with low-energy ions, Thin Solid Films 241 (1994) $167-170$. 